\title{
Apoptotic and anti-angiogenic effects of Pulsatilla koreana extract on hepatocellular carcinoma
}

\author{
SANG-WON HONG ${ }^{*}$, KYUNG HEE JUNG ${ }^{*}$, HEE-SEUNG LEE, MYUNG-JOO CHOI, \\ HONG-MEI ZHENG, MI KWON SON, GUANG-YONG LEE and SOON-SUN HONG \\ Department of Biomedical Sciences, College of Medicine, Inha University, 3-ga, \\ Sinheung-dong, Jung-gu, Incheon 400-712, Republic of Korea
}

Received July 15, 2011; Accepted August 30, 2011

DOI: 10.3892/ijo.2011.1204

\begin{abstract}
Chemoprevention through the use of food and plants has emerged as a novel approach to control various malignancies including cancer. Pulsatilla koreana extract (PKE) has been used to treat malaria and dysentery. The functions and effect of PKE in cancer treatment have been reported but with less information. In this study, we investigated the effect of PKE on the progression of hepatocellular carcinoma (HCC) cells and its mechanism. PKE strongly suppressed the growth of HCC cells in a dose-dependent manner. Apoptosis by PKE was observed by DAPI and TUNEL staining and accompanied with increases of cleaved PARP and caspase-3 in Huh-7 cells. Also, PKE decreased the expression of hypoxia-inducible factor (HIF-1 $\alpha$ ) and vascular endothelial growth factor (VEGF), and inhibited tube formation and migration of human umbilical vein endothelial cells (HUVECs). In addition, PKE potently suppressed in vivo neovascularization in a mouse Matrigel plug assay. Furthermore, in vivo study showed that PKE significantly inhibited tumor growth in a mouse xenograft model, and induced apoptosis by increasing the cleaved PARP and caspase- 3 . The expressions of Ki-67, VEGF, and CD31 in the tumor tissue were decreased by the treatment of PKE. Taken together, our study demonstrates that PKE not only induced apoptosis but also inhibited cell growth and angiogenesis of human HCC. We suggest that PKE is an effective chemotherapeutic candidate for cancer therapy against HCC.
\end{abstract}

\section{Introduction}

Hepatocellular carcinoma (HCC) is one of the most malignant health problems in the world and its effect on mortality has

Correspondence to: Dr Soon-Sun Hong, Department of Biomedical Sciences, College of Medicine, Inha University, 3-ga, Sinheung-dong, Jung-gu, Incheon 400-712, Republic of Korea

E-mail: hongs@inha.ac.kr

*Contributed equally

Key words: Pulsatilla koreana extract, apoptosis, angiogenesis, cancer, hepatocellular carcinoma been increasing over the past few decades in Europe, United States and East Asia $(1,2)$. Although surgical resection and orthotopic liver transplantation are feasible options for therapy in HCC patients with small tumor, patients with advanced HCC are not candidates for these therapies because this approach is often unsatisfactory (3). Thus, the search for additional effective strategies is necessary. One of the most recent and complementary approaches to control cancer is preventive intervention by non-toxic natural extracts or compounds, which target more events related to carcinogenesis and thereby reduce overall cancer risk $(4,5)$. In this regard, plant extracts have been given attention and considered an important class on developing new anti-cancer agents against HCC.

Recently, naturally occurring extracts from plants have been investigated for their therapeutic potential in various cancers (6-8). Pulsatilla koreana extract (PKE) is a traditional Korean herbal medicine, which has been used to treat amoebic dysentery and malaria (9). Numerous studies were reported that some constituents of PKE can lower blood pressure and exhibit anti-inflammatory effects as well as anti-acne activities against aerobic bacteria and fungi $(10,11)$. Also, PKE has been found to have prominent neuroprotective abilities to reverse scopolamine-induced cognitive impairment in rats (10). In addition, several studies have been reported that components of PKE were effective in preventing cancers cells including melanoma and ovarian cancer $(12,13)$.

Despite the chemotherapeutic effects of PKE, the anticancer effect of PKE against HCC has not yet been reported. Also, the mechanisms of the observed chemopreventive and anti-cancer activity by PKE are still unclear, and more information is needed to explain these effects. In this study, therefore, we investigated the effect of PKE on apoptosis and angiogenesis of HCC, which has been directed in the pathogenesis of cancer $(14,15)$.

\section{Materials and methods}

Extraction of PKE. Roots of Pulsatilla koreana were collected from Kyeryong mountain near Daejeon, Korea. The powdered roots of Pulsatilla koreana (50 g) were extracted three times with $50 \%$ aqueous $\mathrm{EtOH}(500 \mathrm{ml})$, and the resulting extracts were combined and concentrated in vacuo to yield a light brown residue. The residue was suspended in acetone $(300 \mathrm{ml})$, and 
centrifuged, and the resulting supernatant was removed to give a brown precipitate. The precipitate was poured into water and filtered to remove the insoluble portion. The filtrate was concentrated to give a brown mass.

Cells and materials. Human HCC cell lines Huh-7 and HepG2 were purchased from JCRB (Shinjuku, Japan), and a normal liver cell line HL-7702 was purchased from the Shanghai Institute of Cell Biology (Shanghai, China). Huh-7 cells were cultured in Roswell Park Memorial Institute Media 1640 (RPMI-1640) and HepG2 cells were cultured in Dulbecco's modified Eagle's medium (DMEM), supplemented with $10 \%$ fetal bovine serum (FBS) and $1 \%$ penicillin/streptomycin. FBS, cell culture media, penicillin-streptomycin, and all other agents used in cell culture studies were purchased from Invitrogen ${ }^{\mathrm{TM}}$ (Gibco, NY). Cultures were maintained at $37^{\circ} \mathrm{C}$ in a $\mathrm{CO}_{2}$ incubator with a controlled humidified atmosphere composed of $95 \%$ air and $5 \% \mathrm{CO}_{2}$. Human umbilical vein endothelial cells (HUVECs) were grown in gelatin coated $75-\mathrm{cm}^{2}$ flasks in a M199 medium containing $20 \mathrm{ng} / \mathrm{ml}$ basic fibroblast growth factor (bFGF), $100 \mathrm{U} / \mathrm{ml}$ heparin and $20 \% \mathrm{FBS}$ at $37^{\circ} \mathrm{C}$. 3-(4,5-dimethylthiazol-2-yl)-2,5-diphenyl tetrazolium bromide (MTT) and proteinase K were purchased from Sigma-Aldrich (St. Louis, MO). RNase A was purchased from Qiagen (Valencia, CA).

Measurement of cell proliferation. Cell viability was performed through an MTT assay. Briefly, Huh-7 and HepG2 cells were plated at a density of $3-5 \times 10^{3}$ cells/well in 96 -well plates for $24 \mathrm{~h}$. The medium was removed, and cells were treated with either DMSO as a control or various concentrations of PKE. After the cells were incubated for $48 \mathrm{~h}, 20 \mu \mathrm{l}$ MTT solutions ( $2 \mathrm{mg} / \mathrm{ml}$ ) were added to each well for another $4 \mathrm{~h}$ at $37^{\circ} \mathrm{C}$. The formazan crystals that formed were dissolved in DMSO (200 $\mu \mathrm{l} /$ well) by constant shaking for $5 \mathrm{~min}$. The plate was then read on a microplate reader at $540 \mathrm{~nm}$. Three replicate wells were used for each analysis.

Western blot analysis. Cells were washed three times with icecold phosphate buffered saline before lysis. Cells were lysed with buffer containing $1 \%$ Triton X-100, 1\% Nonidet P-40, and the following protease and phosphatase inhibitors: aprotinin (10 mg/ml), leupeptin (10 mg/ml) (ICN Biomedicals, AsseRelegem, Belgium), phenylmethylsulfonyl fluoride (1.72 mM), $\mathrm{NaF}(100 \mathrm{mM}), \mathrm{NaVO}_{3}(500 \mathrm{mM})$, and $\mathrm{Na}_{4} \mathrm{P}_{2} \mathrm{O}_{7}(500 \mathrm{mg} / \mathrm{ml})$ (Sigma-Aldrich). Equal amounts of protein were separated by $10 \%$ sodium dodecyl sulfate-polyacrylamide (SDS) gel electrophoresis, transferred onto nitrocellulose membranes and the protein transfer was checked by Ponceau S staining (Sigma-Aldrich). Immunostaining of the blots was performed using the primary antibodies, followed by secondary antibodies conjugated to horseradish peroxidase and detection by enhanced chemiluminescence reagent (ELPS, Seoul, Korea). Primary antibodies were monoclonal antibodies: antiHIF-1 $\alpha$ (BD Biosciences, San Jose, CA), anti-VEGF (Santa Cruz Biotechnology, Santa Cruz, CA), anti-Bax (Santa Cruz Biotechnology), anti-cleaved caspase-3, anti-cleaved PARP (Cell Signaling Technologies, Danvers, MA). The secondary antibodies were purchased from Amersham Biosciences. The bands were visualized with the ECL plus system (Amersham Pharmacia Biotech, Inc., Piscataway, NJ).
DAPI staining and TUNEL assay. Huh-7 cells were plated onto $18 \mathrm{~mm}$ cover glasses in RPMI-1640 medium at $70 \%$ confluence for $24 \mathrm{~h}$. The cells were then treated with PKE at $100 \mu \mathrm{g} /$ $\mathrm{ml}$ for $24 \mathrm{~h}$. They were fixed in $2 \%$ ice-cold para-formaldehyde (PFA), washed with PBS and then stained with $2 \mu \mathrm{g} / \mathrm{ml}$ of 4,6-diamidino-2-phenylindole (DAPI) for $20 \mathrm{~min}$ at $37^{\circ} \mathrm{C}$. The DAPI stained cells were examined under a fluorescent microscope analyzing (fluorescent) nuclear fragmentation. Terminal deoxynucleotidyl transferase-mediated nick end labeling (TUNEL) was performed following the manufacturer's protocol for TUNEL kit (Chemicon, Temecula, CA).

Tube formation assay. Matrigel $(200 \mu \mathrm{l})(10 \mathrm{mg} / \mathrm{ml})(\mathrm{BD}$ Biosciences, New Jersey) was polymerized for $30 \mathrm{~min}$ at $37^{\circ} \mathrm{C}$. HUVECs were suspended in M199 (5\% FBS) medium at a density of $2.5 \times 10^{5}$ cells $/ \mathrm{ml}$, and $0.2 \mathrm{ml}$ of cell suspension was added to each well coated with Matrigel, together with or without the indicated concentrations of PKE for $14 \mathrm{~h}$. The morphological changes of the tube formation were observed under a phase-contrast microscope and photographed at x200 magnification.

Migration assay. HUVECs, plated on $60 \mathrm{~mm}$ diameter culture dishes at $90 \%$ confluence, were wounded with a $2 \mathrm{~mm}$ razor blade and marked at the injury line. After wounding, the peeled off cells were removed with a serum-free medium and further incubated in M199 with 5\% serum, $1 \mathrm{mM}$ thymidine (SigmaAldrich) and/or PKE (100 $\mu \mathrm{g} / \mathrm{ml})$. HUVECs were allowed to migrate for $24 \mathrm{~h}$ and were rinsed with a serum-free medium, followed by fixing with absolute methanol and staining with Giemsa (Sigma-Aldrich). Migration was quantitated by counting the number of cells that moved beyond the reference line.

Matrigel plug assay. The BALB/c mice (6-week) were injected subcutaneously with $600 \mu \mathrm{l}$ of Matrigel (BD Bioscience) containing concentrated VEGF $(50 \mathrm{ng} / \mathrm{ml})$ with heparin (15 U/ $/ \mu \mathrm{l})$, and either PKE $(100 \mu \mathrm{g} / \mathrm{ml})$ or PBS. After 7 days, the mice were sacrificed, and the Matrigel plugs were recovered, fixed with $4 \%$ paraformalde in PBS, and embedded in paraffin. A part of each plug was fixed, sectioned, and either stained with Hematoxylin-eosin $(\mathrm{H} \& \mathrm{E})$ or immunostained with the antibody for CD34.

Tumor xenograft study. Male nude mice were obtained from Central Lab. Animal Inc. (Seoul, Korea). Animal care and all experimental procedures were conducted in accordance with the approval and guidelines of the INHA Institutional Animal Care and Use Committee (INHA IACUC) of the Medical School of Inha University (approval ID: 090518-5). The animals were fed standard rat chow and tap water ad libitum, and were maintained under $12 \mathrm{~h}$ dark/light cycle at $21^{\circ} \mathrm{C}$. Male nude mice $(6$ weeks, weighing 22-26 g) were randomly divided into four groups (control, PKE $125 \mathrm{mg} / \mathrm{kg}$, PKE $250 \mathrm{mg} / \mathrm{kg}$, and Sorafenib $10 \mathrm{mg} / \mathrm{kg}$ ). Huh-7 cells were harvested and mixed with PBS (200 $\mu \mathrm{l} / \mathrm{mouse}$ ) and then inoculated into one flank of each nude mouse $\left(5 \times 10^{6}\right.$ of Huh- 7 cells). When the tumors had reached a volume of about $50 \mathrm{~mm}^{3}$, mice were given a daily intraperitoneal injection of PKE (125 and $250 \mathrm{mg} / \mathrm{kg}$, treated group) or the vehicle (200 $\mu \mathrm{l}$ PBS, control group), and orally 
A

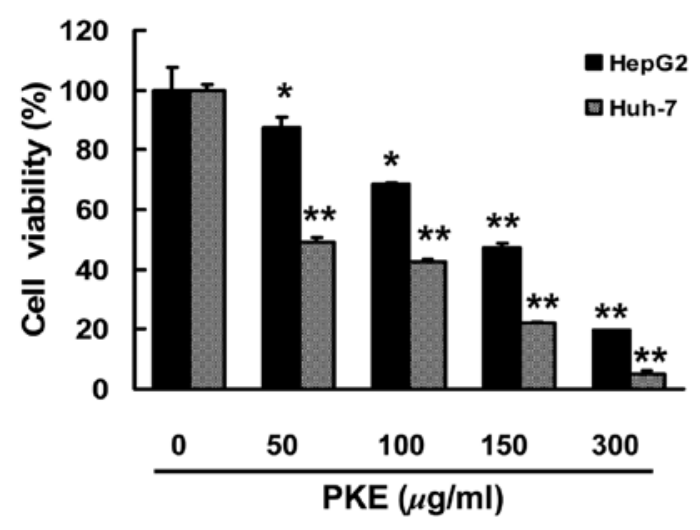

B

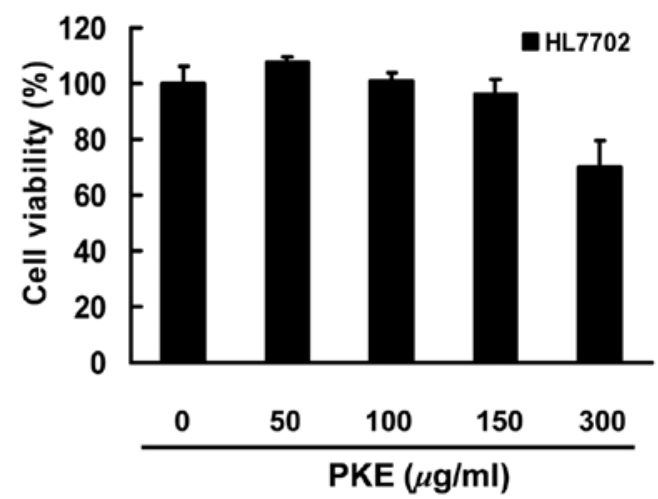

Figure 1. Effect of PKE on the proliferation of HCC and normal liver cells. (A) Cytotoxic effects of PKE on HCC cells (HepG2 and Huh-7) and (B) normal liver cell line (HL-7702) were measured by an MTT assay. HCC and a normal liver cells were seeded in a 96-well culture plates. After incubation for 24 h, the cells were treated with various concentrations of PKE. After incubation for $48 \mathrm{~h}$, they were subjected to the MTT assay. Results are expressed as percent cell proliferation relative to the proliferation of the control. Data are represented as the mean \pm SD from the triplicate wells. ${ }^{*} \mathrm{p}<0.05$ and ${ }^{* *} \mathrm{p}<0.01$ vs. control.

Sorafenib (positive control group) for 16 days. The tumor dimensions were measured twice a week using a digital caliper and the tumor volume was calculated using the formula: $\mathrm{V}=$ length $\mathrm{x}$ width $^{2} \times 0.5$. At the end of the experiment, mice were sacrificed, and tumors were excised and weighed. A part of each tumor was fixed in buffered formalin and the remaining tissue was stored at $-70^{\circ} \mathrm{C}$ for further analysis.

Fluorescence immunohistochemistry. Frozen sections of $10 \mu \mathrm{m}$ were incubated overnight at $4^{\circ} \mathrm{C}$ in 1:100 dilutions of mouse anti-CD31 antibody (Santa Cruz Biotechnology). After washing three times with PBS, detection of CD31 primary antibody was performed using a 1:200 dilution of mouse FITF-labeled secondary antibody (Vector Laboratories, Burlingame, CA). After washing with PBS three times, each slide was occluded with $50 \%$ glycerin buffer and was observed using a confocal laser scanning microscope (Olympus, Tokyo, Japan).

Immunohistochemistry. Immunostaining was performed on $8 \mu \mathrm{m}$-thick sections after deparaffinization. Microwave antigen retrieval was performed in citrate buffer ( $\mathrm{pH}$ 6.0) for $10 \mathrm{~min}$ prior to peroxidase quenching with $3 \% \mathrm{H}_{2} \mathrm{O}_{2}$ in $\mathrm{PBS}$ for $10 \mathrm{~min}$. Sections were then washed in water and preblocked with normal goat or horse serum for $10 \mathrm{~min}$. Next, tissue sections were incubated overnight at $4^{\circ} \mathrm{C}$ in 1:50 dilutions of mouse antiKi-67 and anti-VEGF antibodies (Santa Cruz Biotechnology). The sections were then incubated with biotinylated secondary antibodies (1:200) for $1 \mathrm{~h}$. Following a washing step with PBS, streptodavidin-HRP was applied. Finally, the sections were developed with diaminobenzidine tetrahydrochloride substrate for $10 \mathrm{~min}$, and counterstained with hematoxylin. At least three random fields of each section were examined at a magnification of x 200 and analyzed by a computer image analysis system (Media Cybernetics, Silver Spring, MD).

Statistical analysis. Data are expressed as the mean $\pm \mathrm{SD}$, and statistical analysis was performed using ANOVA and an unpaired Student's t-test. A p $\leq 0.05$ was considered statistically significant. Statistical calculations were performed using SPSS software for Windows operating system (Version 10.0; SPSS, Chicago, IL).

\section{Results}

PKE inhibits growth of hepatocellular carcinoma cells. We first examined the effect of PKE on the growth and viability of two HCC cell lines (HepG2 and Huh-7 cells). HCC cells were exposed to four concentrations from 50 to $300 \mu \mathrm{g} / \mathrm{ml}$ of PKE for $48 \mathrm{~h}$. Our result showed that cell growth was inhibited by PKE treatment in a dose-dependent manner (Fig. 1A). In HepG2 cells, PKE treatment at 100-300 $\mu \mathrm{g} / \mathrm{ml}$ concentrations decreased the total cell number from $20 \%$ to $70 \%$, respectively. Of special note, PKE decreased cell growth by about $50-90 \%$ at the designated concentration in Huh-7 cells. As Huh-7 cells were more sensitive to PKE-mediated inhibition of cell growth and proliferation, we chose this cell line for further experiments. To predict possible side effects of PKE in normal hepatocytes, we also treated PKE to HL-7702, a normal human liver cell line. Cell viability with PKE was not changed in HL-7702 in concentrations of 50-150 $\mu \mathrm{g} / \mathrm{ml}$ although PKE of $300 \mu \mathrm{g} / \mathrm{ml}$ showed a slight change in cell viability.

Effects of PKE on apoptotic cell death in Huh-7 cells. To identify the apoptotic effect of PKE in Huh-7 cells, we performed analysis through DAPI and TUNEL staining. When PKE $(100 \mu \mathrm{g} / \mathrm{ml})$ was treated to Huh-7 cells, the cells presented morphological features of apoptotic cells, such as bright nuclear condensation, DNA fragmentation, and perinuclear apoptotic bodies by DAPI staining (Fig. 2A). Data of the TUNEL staining also exhibited PKE induced apoptosis by causing DNA strand breaks. Next, we investigated the activation of Bax, caspase-3, and the cleavage of PARP by Western blotting on $48 \mathrm{~h}$ after PKE treatment. PKE led the activation of Bax and the cleaved caspase- 3 and subsequently the cleavage of PARP in Huh-7 cells in a dose-dependent manner (Fig. 2B). These results showed that PKE could induce cell apoptosis in Huh-7 HCC cells. 
A
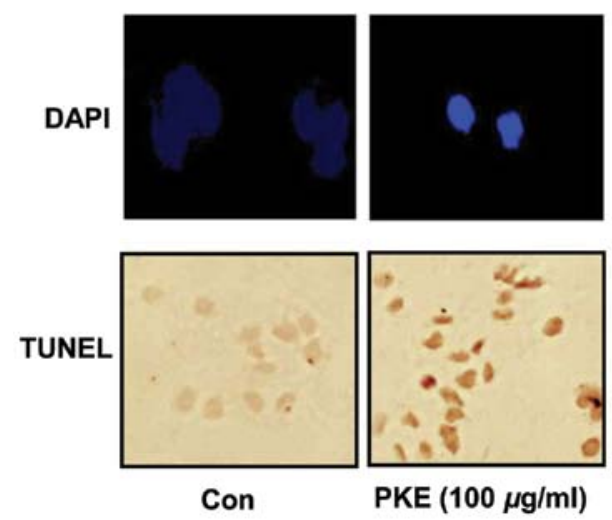

B

$\operatorname{PKE}(\mu \mathrm{g} / \mathrm{ml}) \quad 0 \quad 50 \quad 100 \quad 150 \quad 300$

Bax

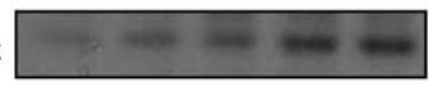

Cleaved caspase 3

Cleaved PARP

$\beta$-actin
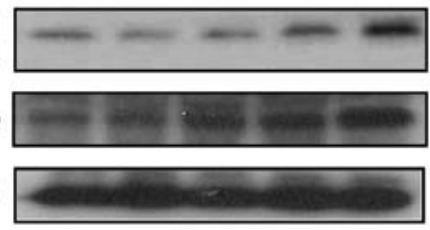

Figure 2. Effect of PKE on apoptosis of Huh-7 cells. (A) The induction of apoptosis by PKE was conducted by TUNEL and DAPI staining, which were photographed at $\times 200$ magnification. (B) The expression of Bax, the cleaved PARP, and cleaved caspase- 3 were determined by Western blotting in cells treated with $\mathrm{PKE}$ at the indicated doses for $48 \mathrm{~h}$.

A

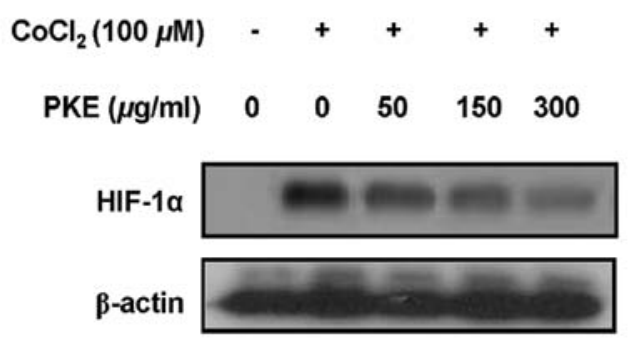

B

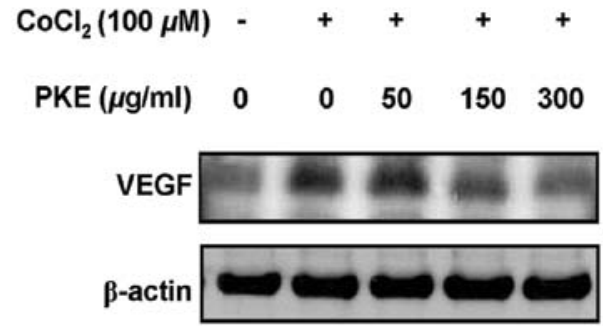

$\operatorname{PKE}(\mu \mathrm{g} / \mathrm{ml})$

C

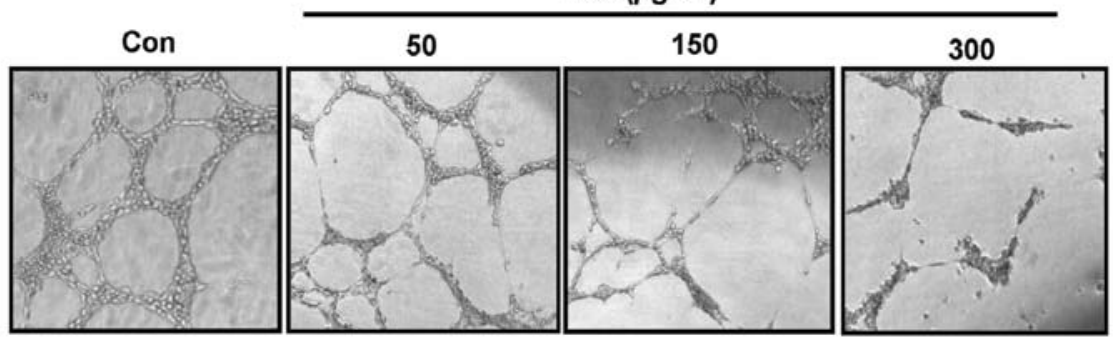

D

$2 \%$ FBS

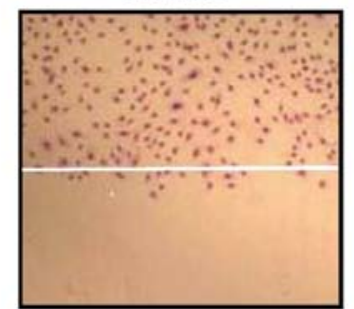

$5 \%$ FBS

$5 \%$ FBS+PKE $(100 \mu \mathrm{g} / \mathrm{ml})$
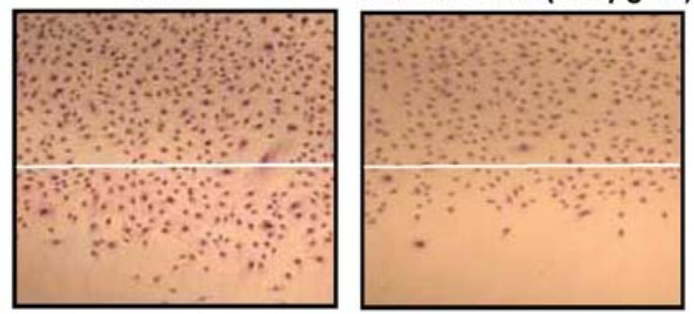

Figure 3. Effect of PKE on angiogenesis of Huh-7 cells. (A) Expression of HIF-1 $\alpha$ and (B) VEGF by PKE in hypoxia-induced Huh-7 cells (CoCl $\left.{ }_{2}, 100 \mu \mathrm{M}\right)$. (C) Effects of PKE on tube formation in vitro. HUVECs were plated on Matrigel ( $200 \mu \mathrm{l} /$ well) and treated with various concentrations of PKE. Capillary tube formation was assessed after $14 \mathrm{~h}$. Tube formation was observed under a phase-contrast microscope and photographed at x400 magnification. (D) Effects of PKE on migration in vitro. HUVECs were plated at $90 \%$ confluence and a scratched was made with a razor blade. After wounding, the cells were washed with a serum-free medium and incubated in M199 with 5\% FBS and $5 \mathrm{ng} / \mathrm{ml} \mathrm{bFGF,} 1 \mathrm{mM}$ thymidine and/or PKE (100 $\mu \mathrm{g} / \mathrm{ml})$. M199 medium with 2\% FBS was used as negative control. Data represent the mean of at least three independent experiments done in triplicate. 

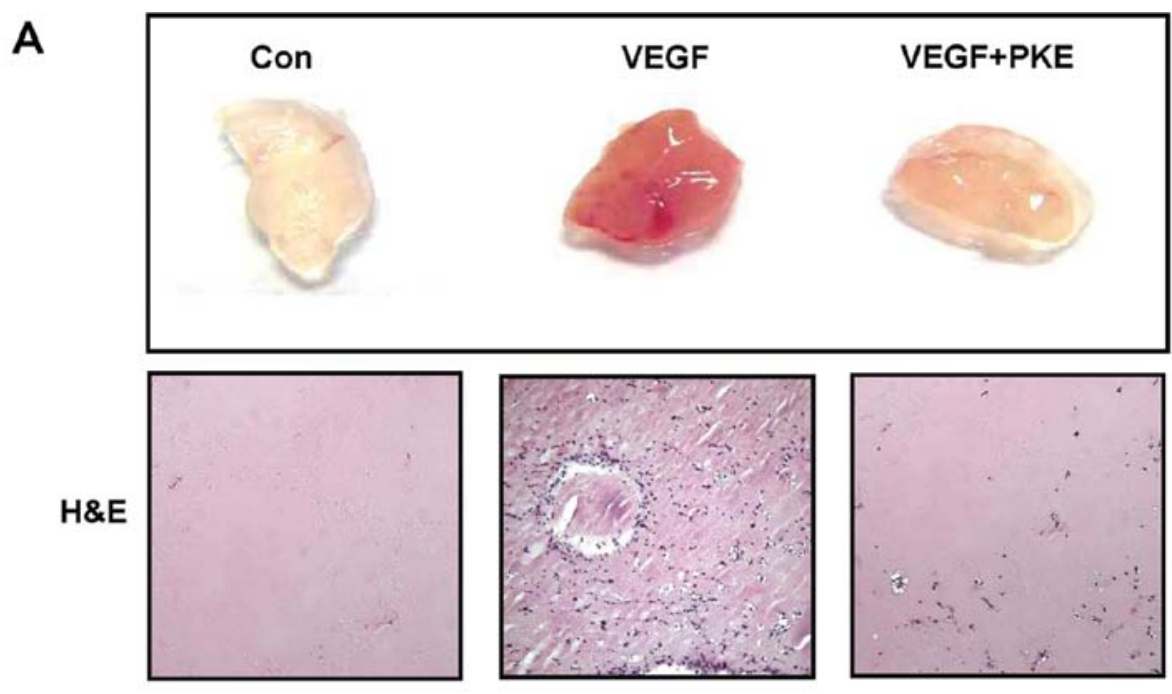

B

DAPI
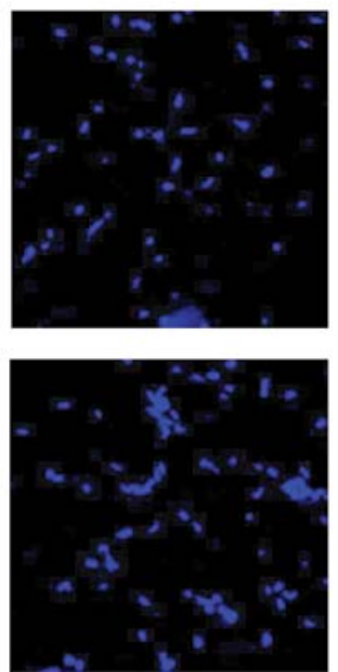

CD 34
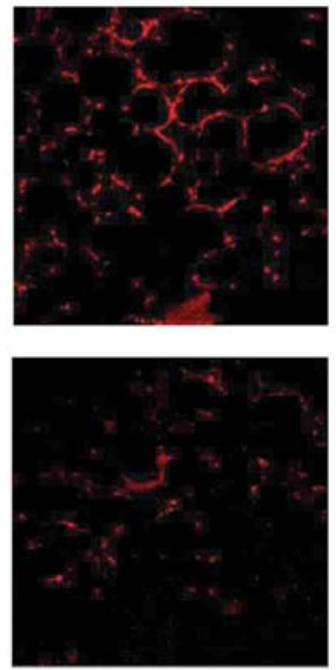

Merge
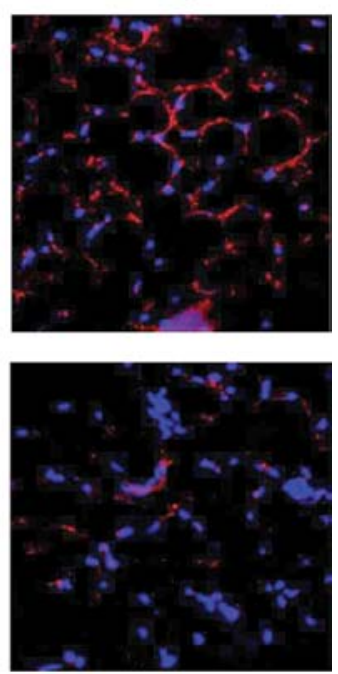

Figure 4. Effect of PKE on Matrigel plug assay in vivo. (A) Matrigel plugs were implanted into mice with VEGF (50 ng/ml) and/or PKE (100 $\mu \mathrm{g} / \mathrm{ml})$, and were photographed to show the extent of vascularisation after 7 days. The plugs were sectioned and stained with H\&E. (B) Endothelial cells in the plug were immunostained with the CD34. The stained plugs were observed by microscopy at x200 and x400 magnification.

Effects of PKE on angiogenesis in Huh-7 cells. HIF-1 $\alpha$ plays a central role in tumor progression and angiogenesis, and is also one of major transcriptional modulators of angiogenic factors such as VEGF. Thus, we examined the effect of PKE on expression of hypoxia induced HIF-1 $\alpha$ and VEGF. Cells were treated with various concentration of PKE under hypoxia mimic condition induced by $\mathrm{CoCl}_{2}(100 \mu \mathrm{M})$ for $6 \mathrm{~h}$. As shown in Fig. 3A, HIF-1 $\alpha$ expression was increased under hypoxic conditions. However, PKE treatment at 50 to $300 \mu \mathrm{g} / \mathrm{ml}$ inhibited the hypoxiainduced HIF-1 $\alpha$ expression in a dose-dependent manner. Also, VEGF expression was increased under the hypoxia conditions, whereas treatment of PKE inhibited VEGF expression at 150 to $300 \mu \mathrm{g} / \mathrm{ml}$ (Fig. 3B). In addition, the anti-angiogenic potential of PKE was examined using HUVECs. From an in vitro tube formation assay, we observed that PKE inhibited the formation of vessel-like structures, consisting of the elongation and align- ment of the cells at the indicated concentrations (Fig. 3C). Cell migration is critical for endothelia cell to form blood vessels in angiogenesis and is necessary for tumor growth and metastasis. Thus, we carried out a wound migration assay to examine the effect of PKE on cell migration. When the endothelial cells were wounded and incubated in media with $5 \% \mathrm{FBS}$ and $1 \mathrm{mM}$ thymidine in the presence of PKE $(100 \mu \mathrm{g} / \mathrm{ml})$ for $24 \mathrm{~h}$, the wound after PKE treatment was remarkably unable to heal (Fig. 3D). Our results therefore indicate that PKE could prevent tube formation and migration of endothelial cell, suggesting that PKE has a potent anti-angiogenic property.

Inhibition of tumor growth by PKE in mouse xenograft model. Based on our results demonstrating a strong efficacy of PKE against Huh-7 cells, we next examined the in vivo efficacy of PKE against the Huh-7 cell xenograft in nude mice (Fig. 4). 
A

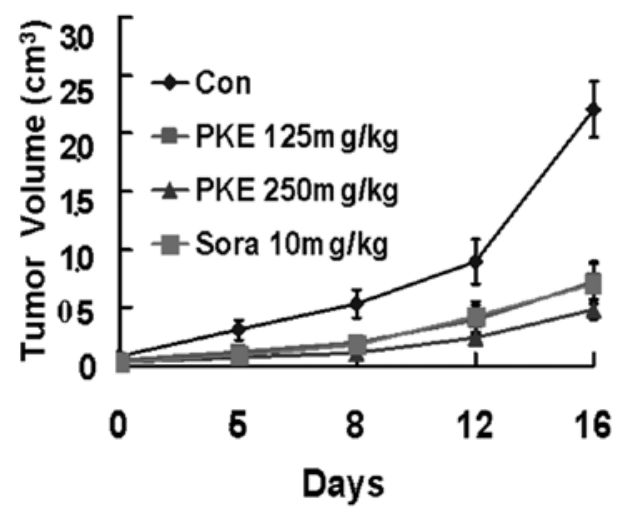

B

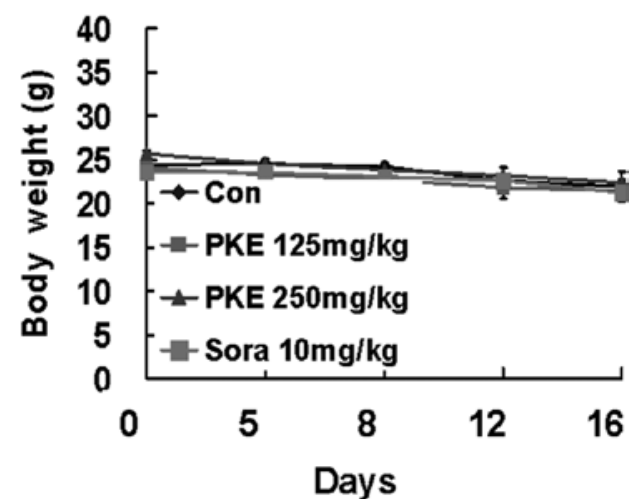

C
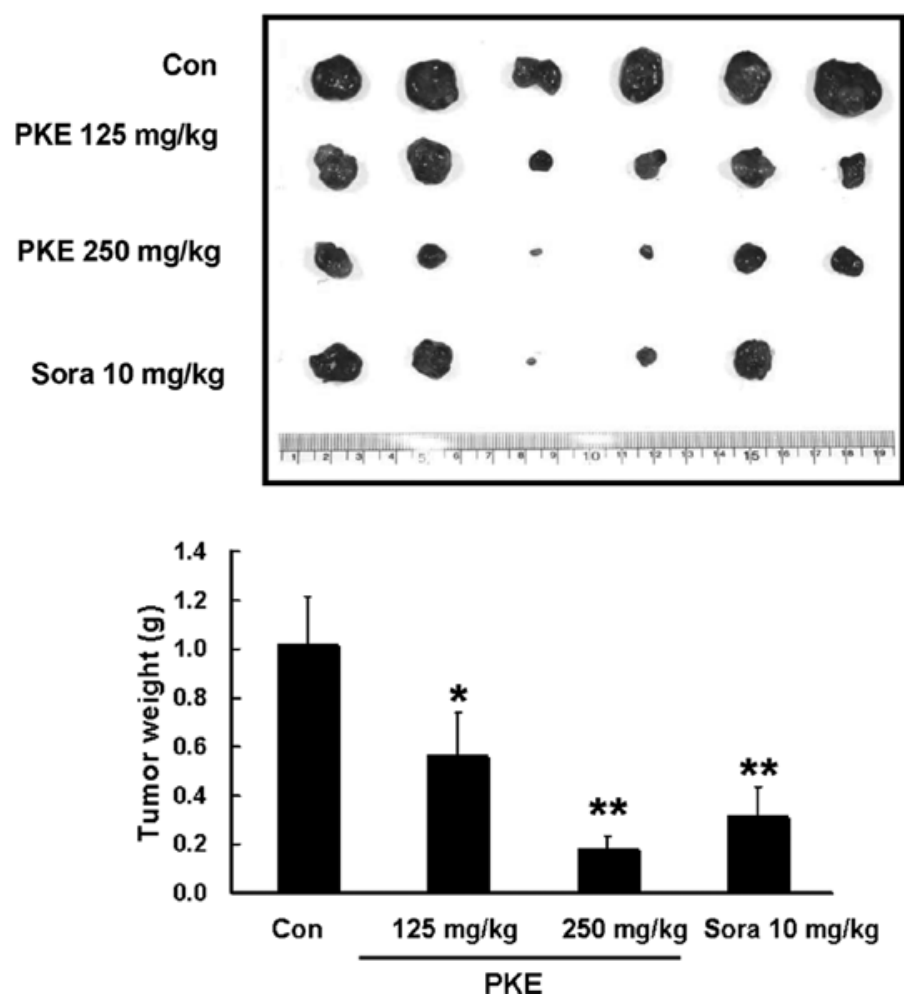

Figure 5. In vivo effect of PKE on HCC mouse xenograft model. (A) Tumor growth of Huh-7 cells xenograft in nude mice. (B) Average body weight of nude mice. All mice were implanted by subcutaneous injection of Huh-7 cells $\left(5 \times 10^{6}\right.$ cells $/ 200 \mu 1$ PBS) on the flank. The treatment of PKE had little difference in the body weight compared with the control group. (C) Tumor size and weight in Huh-7 mouse xenograft. Data is represented by mean \pm SD $(n=5$ or 6$)$. ${ }^{*}<0.05$ and ${ }^{* *} \mathrm{p}<0.01$ vs. control. Sora, Sorafenib.

First, we observed that there was no difference of body weight in groups of PKE treatment compared with the control group, indicating that PKE has a low toxicity to mice at the curative dose. As shown in Fig. 5A, PKE induced dose-dependent inhibition of tumor growth at doses of 125 or $250 \mathrm{mg} / \mathrm{kg}$ for 16 days compared with the control group. PKE administration at doses of 125 and $250 \mathrm{mg} / \mathrm{kg}$ resulted in significant reduction of tumor volume (67\% and 79\%, respectively) of nude mice. Especially, a PKE of $250 \mathrm{mg} / \mathrm{kg}$ dose suppressed tumor growth to an extent comparable to or greater than $10 \mathrm{mg} / \mathrm{kg}$ of Sorafenib, an oral drug for advanced HCC (Fig. 5A). Consistent with this observation, the weight of tumors isolated from PKE-treated groups was significantly decreased $55 \%$ and $17 \%$ at PKE doses of 125 and $250 \mathrm{mg} / \mathrm{kg}$, respectively compared with the control (Fig. 5C, $\mathrm{p}<0.05)$. Our results demonstrate in vivo anti-tumor efficacy of PKE against HCC without any apparent sign of toxicity.

Inhibition of angiogenesis and proliferation with induction of apoptosis by PKE in HCC xenograft model. In H\&E staining, we observed that there was a greater degree of tumor apoptosis and necrosis in the PKE-treated group compared with the control group. Also, the results of Fig. 6A showed that PKE decreased the expression of the cell proliferation marker Ki-67 in tumor tissues. Meanwhile, tumor cells have been known to 

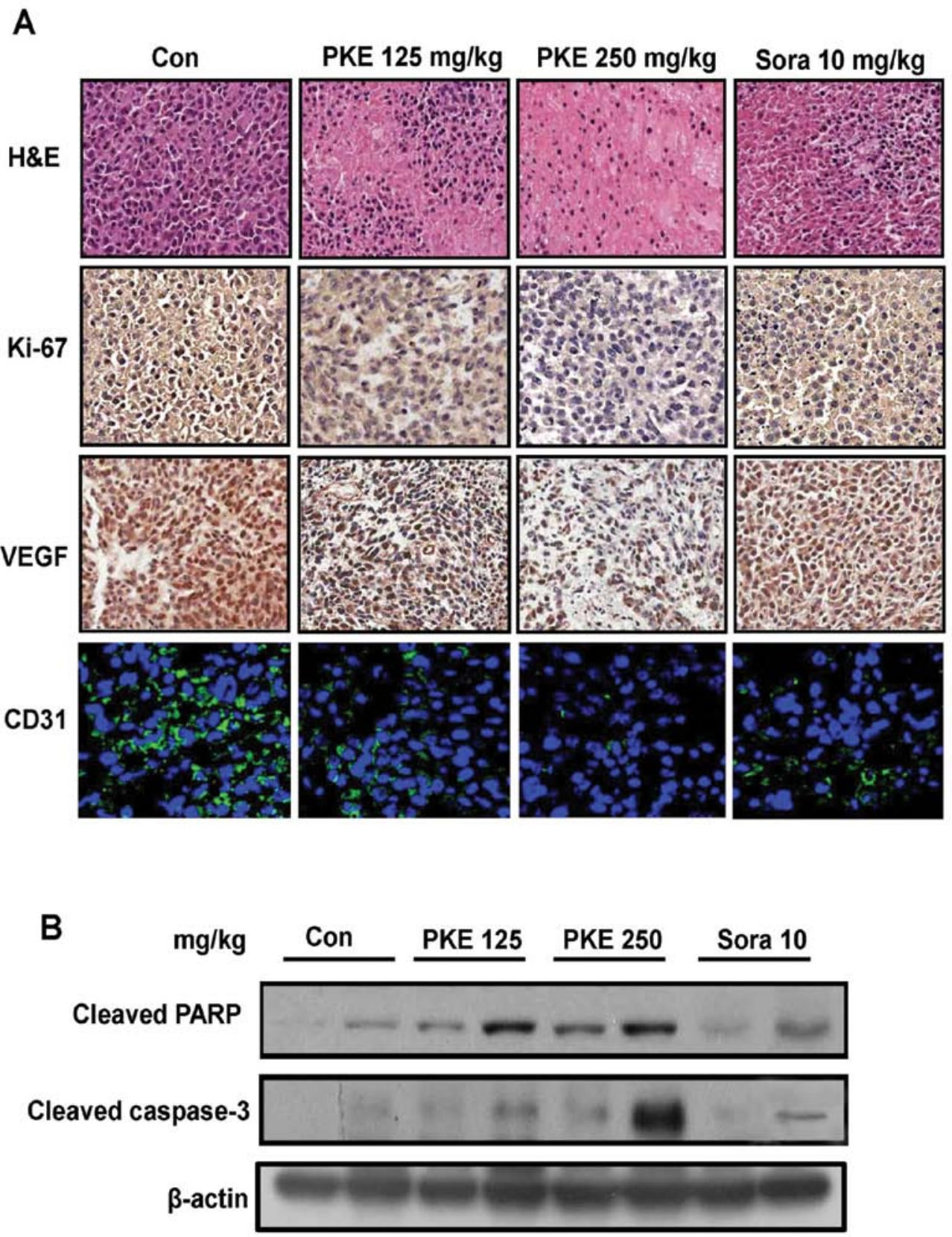

Figure 6. In vivo effect of PKE on proliferation, angiogenesis, and apoptosis of HCC xenografts. (A) Tumors were excised and processed for immunostaining for Ki-67, VEGF, and CD31 with H\&E staining. Original magnification, x400. (B) The expression of the cleaved PARP, the cleaved caspase-3 was assayed by Western blotting in tumor tissue. Sora, Sorafenib.

produce and secret VEGF for neovascularization of the tumor. Our cell culture study showed that PKE inhibited VEGF expression (Fig. 3B). Therefore, to assess the in vivo effect of PKE on angiogenesis, we investigated the expression of VEGF and CD31 in tumor tissues. In microscopic analysis, the expression of both VEGF and CD31 showed a strong decrease in the PKE treated group compared with the control group. In addition, the apoptotic effect of PKE on HCC tumor tissues was identified by expression of the cleaved PARP and caspase-3 (Fig. 6B). Interestingly, the effect of PKE on apoptosis was more potent than that of Sorafenib. The increased expression of the cleaved caspase- 3 and PARP in vivo was quite similar to the results of the in vitro study.

\section{Discussion}

Many plants are main sources of drugs that have successfully made their way into the clinic. Indeed, about $70 \%$ of all drugs used in chemotherapy are either products of natural origin or may be based on their pharmachophores (16). Therefore, chemoprevention approach using natural products or extracts could be more practical and effective in the fight against cancer. In this study, we investigated anti-cancer efficacy and associated mechanisms of PKE against HCC cells in vitro, and expanded into an in vivo $\mathrm{HCC}$ xenograft animal model. Our study revealed that PKE inhibited cell or tumor growth and induced apoptosis in both Huh-7 cells and the xenograft animal model. In addition, 
PKE suppressed angiogenesis by decreasing the expression of HIF- $1 \alpha$ and VEGF.

Apoptosis plays a pivotal role in preventing cancer. If a cell is unable to undergo apoptosis because of a mutation or a biochemical inhibition, it can continue dividing and develop to become a tumor (17). Caspase-3 is the main key component of apoptosis, being responsible either partially or totally for the proteolytic cleavage of many key proteins (18). In addition, caspase-3 cleaves PARP, inhibiting PARP activity during apoptosis. Thus, we first decided to test the anti-cancer effects of PKE by the mechanism of apoptosis in Huh-7 cells. In this study, we observed that PKE increased the expression of the cleaved caspase-3 and PARP, leading to apoptotic cell death. As the in vitro results, the administration of PKE in doses of 125 and $250 \mathrm{mg} / \mathrm{kg}$ increased the expression of cleaved caspase-3 and PARP. These apoptotic effects of PKE were confirmed by the results that PKE increased DNA fragmentation, nuclear condensation, and cell morphology changes by TUNEL and DAPI staining. These results implied that apoptosis by PKE may be an important factor in the suppression of tumor growth.

Tumor angiogenesis is a complex interrelated multistep process. Inhibition of any step in this process may lead to the disruption of angiogenesis and can serve as a potential antitumor therapy. Recently, it has been reported that the angiogenic response increases during hepatocarcinogenesis, and targeting VEGF significantly attenuated HCC development and metastasis (19). HIF-1 $\alpha$ is also known as a target regulator of angiogenesis along with VEGF in various types of cancers $(20,21)$. Since inhibition of VEGF could be due to inactivation of HIF-1 $\alpha, \mathrm{VEGF} /$ HIF-1 $\alpha$ may provide a potential target for a novel therapeutic strategy against HCC. Our results showed that PKE inhibited expression of HIF-1 $\alpha$ and VEGF under hypoxia conditions induced by $\mathrm{CoCl}_{2}$ in Huh-7 cells. In the in vivo study, $\mathrm{PKE}$ at 125 and $250 \mathrm{mg} / \mathrm{kg}$ also inhibited angiogenesis by decreasing the expression of VEGF and CD31. As in this point, inhibition of tumor growth by PKE seems to affect angiogenic factors such as VEGF. Indeed, natural products including green tea and ginkgo biloba extracts have been reported to show anti-angiogenesis effect through inhibition of VEGF $(22,23)$. In addition, the in vivo anti-angiogenic effects of PKE was supported by decreased expression of CD34, a microvessel endothelial cell marker, in a VEGF-mediated Matrigel plug assay in parallel with inhibition of HUVEC cell migration and tube formation, indicating that PKE inhibited angiogenesis through not only VEGF but also targeting endothelial cells directly. More importantly, in vivo experiment, PKE showed potent inhibition of tumor growth by inducing apoptosis and anti-angiogenesis. Interestingly, PKE of a $250 \mathrm{mg} / \mathrm{kg}$ dose suppressed more tumor growth than $10 \mathrm{mg} / \mathrm{kg}$ of Sorafenib, which is currently used as the standard care drug in patients with advanced HCC. It has been reported that Sorafenib exhibited side effect such as handfoot skin reactions, diarrhea, and hypertension in HCC patient (24). In this regard, it seems that a natural product such as PKE has great potential to be an anti-cancer agent.

In conclusion, the mechanisms of the anti-cancer effects by PKE are not fully elucidated, but it is thought likely that PKE induces apoptosis by increasing activity of the cleaved caspase-3 and PARP, and inhibits angiogenesis through inhibition of VEGF. These findings suggest that PKE may be a potential candidate for cancer therapy against HCC. Further studies are needed to investigate which component(s) of PKE exhibits the anti-cancer effect(s) by being involved in apoptosis and anti-angiogenesis, which would facilitate PKE-mediated chemotherapy for HCC.

\section{Acknowledgements}

This study was supported by the Korean Health Technology R\&D Project (A101185) and the National R\&D Program for Cancer Control (1020250), Ministry of Health \& Welfare, and National Research Foundation of Korea (NRF) funded by the Ministry of Education, Science and Technology (2011-0005255), and SBPharmaceutical IND. Co.

\section{References}

1. Gomaa AI, Khan SA, Toledano MB, Waked I and TaylorRobinson SD: Hepatocellular carcinoma: epidemiology, risk factors and pathogenesis. World J Gastroenterol 14: 4300-4308, 2008.

2. Chrisey L, Rossi J and Sarver N: Meeting Report. Ribozymes: progress and prospects of catalytic RNA as therapeutic agents. Antisense Res Dev 1: 57-63, 1991.

3. El-Serag HB, Marrero JA, Rudolph L and Reddy KR: Diagnosis and treatment of hepatocellular carcinoma. Gastroenterology 134: 1752-1763, 2008.

4. Naithani R, Huma LC, Moriarty RM, McCormick DL and Mehta RG: Comprehensive review of cancer chemopreventive agents evaluated in experimental carcinogenesis models and clinical trials. Curr Med Chem 15: 1044-1071, 2008.

5. Surh YJ: Cancer chemoprevention with dietary phytochemicals. Nat Rev Cancer 3: 768-780, 2003.

6. Velmurugan B, Singh RP, Agarwal R and Agarwal C: Dietaryfeeding of grape seed extract prevents azoxymethane-induced colonic aberrant crypt foci formation in fischer 344 rats. Mol Carcinog 49: 641-652, 2010

7. Jiang XY, Qian LP, Zheng XJ, Xia YY, Jiang YB and Sun da Y: Interventional effect of Ginkgo biloba extract on the progression of gastric precancerous lesions in rats. J Dig Dis 10: 293-299, 2009.

8. Gutierrez-Orozco F, Stephens BR, Neilson AP, Green R, Ferruzzi MG and Bomser JA: Green and black tea inhibit cytokineinduced IL- 8 production and secretion in AGS gastric cancer cells via inhibition of NF-kappaB activity. Planta Med 76: 1659-1665, 2010.

9. Bang KH: The medicinal plant of Korea. Kyo-Hak Press, p139, 1999.

10. Cho SC, Sultan MZ and Moon SS: Anti-acne activities of pulsaquinone, hydropulsaquinone, and structurally related 1,4-quinone derivatives. Arch Pharm Res 32: 489-494, 2009.

11. Martin ML, San Roman L and Dominguez A: In vitro activity of protoanemonin, an antifungal agent. Planta Med 56: 66-69, 1990.

12. Bang SC, Lee JH, Song GY, Kim DH, Yoon MY and Ahn BZ: Antitumor activity of Pulsatilla koreana saponins and their structure-activity relationship. Chem Pharm Bull (Tokyo) 53: 1451-1454, 2005.

13. Kim Y, Kim SB, You YJ and Ahn BZ: Deoxypodophyllotoxin; the cytotoxic and antiangiogenic component from Pulsatilla koreana. Planta Med 68: 271-274, 2002.

14. Zhang T, Sun HC, Zhou HY, Luo JT, Zhang BL, Wang P, Wang L, Qin LX, Ren N, Ye SL, Li Q and Tang ZY: Interferon alpha inhibits hepatocellular carcinoma growth through inducing apoptosis and interfering with adhesion of tumor endothelial cells. Cancer Lett 290: 204-210, 2010.

15. Kuang DM, Zhao Q, Wu Y, Peng C, Wang J, Xu Z, Yin XY and Zheng L: Peritumoral neutrophils link inflammatory response to disease progression by fostering angiogenesis in hepatocellular carcinoma. J Hepatol 54: 948-955, 2011.

16. Cragg GM, Newman DJ and Yang SS: Natural product extracts of plant and marine origin having antileukemia potential. The NCI experience. J Nat Prod 69: 488-498, 2006.

17. O'Connor L, Huang DC, O'Reilly LA and Strasser A: Apoptosis and cell division. Curr Opin Cell Biol 12: 257-263, 2000.

18. Yuan J and Horvitz HR: A first insight into the molecular mechanisms of apoptosis. Cell 116 (Suppl. 2): S53-S59, 2004. 
19. Wu XZ: New strategy of antiangiogenic therapy for hepatocellular carcinoma. Neoplasma 55: 472-481, 2008.

20. Bolat F, Haberal N, Tunali N, Aslan E, Bal N and Tuncer I: Expression of vascular endothelial growth factor (VEGF), hypoxia inducible factor 1 alpha (HIF-1alpha), and transforming growth factors beta1 (TGFbeta1) and beta3 (TGFbeta3) in gestational trophoblastic disease. Pathol Res Pract 206: 19-23, 2010.

21. NankoN,TanikawaM,MaseM,FujitaM,TateyamaH,Miyati Tand Yamada K: Involvement of hypoxia-inducible factor-1alpha and vascular endothelial growth factor in the mechanism of development of chronic subdural hematoma. Neurol Med Chir (Tokyo) 49: 379-385, 2009.
22. Sartippour MR, Pietras R, Marquez-Garban DC, Chen HW, Heber D, Henning SM, Sartippour G, Zhang L, Lu M, Weinberg O, Rao JY and Brooks MN: The combination of green tea and tamoxifen is effective against breast cancer. Carcinogenesis 27: 2424-2433, 2006

23. DeFeudis FV, Papadopoulos V and Drieu K: Ginkgo biloba extracts and cancer: a research area in its infancy. Fundam Clin Pharmacol 17: 405-417, 2003.

24. Llovet J, Ricci S and Mazzaferro V: Sorafenib in advanced hepatocellular carcinoma. N Engl J Med 359: 378-390, 2008. 